\title{
Efficient Load Balancing by Adaptive Bypasses for the Migration on the Internet
}

\author{
Yukio Hayashi \\ Japan Advanced Institute of Science and Technology, Ishikawa, Japan
}

\begin{abstract}
We study a dynamic load balancing problem for servers on the Internet as Grid, based on the differences of distributed computing to parallel computing. We propose an adaptive method according to initially assigned load; the optimal migration flow is directly obtained without any iterations, and the conditions of migration for the bottleneck edges are relaxed by the bypasses on a cactus adaptively extended from a spanning tree. Simulation results show that the number of rounds for the migration is decreased under $2 / 3$ for the conventional method, and that the cost with respect to the instability of migration is also decreased in about the half. Thus, the adaptively constructed cactus is considered as a practically efficient topology.
\end{abstract}

\section{Introduction}

On the rapid growth of computer networks, distributed computing has attracted considerable attention. In the efficient distributed computing, load balancing is one of the important issues. The problem is to balance the load of servers (computers in general) on a network such as the Internet. In other words, it is to suppress the idling or over-load states as few as possible by using only local communications between servers. Thus, we should remark that it is different from the load balancer for Web-accesses at the end of switch level (Layer 4 or 5) based on the DNS round robin [1] or for a centralized master/slave system.

Load balancing algorithms have been so far developed for parallel computers, whose processor is corresponding to the server in a distributed system. For example, to only locally balance the differences of load between neighbors, initiation methods are triggered by a processor with load level under or over a certain threshold. The former is called "receiver-initiation," and the later "senderinitiation" [7. They are very simple but ad hoc. In addition, the global balancing is not guaranteed, and the setting of threshold is generally difficult.

On the other hand, the most popular approach to avoid the returns of migration is based on two phases: 1) calculation of the migration flow on each connection, 2) migration of the load between the nearest-neighbors in run-time. As the typical strategies [10, there are dimension exchange (DE) and diffusion (DF) methods. With the DE method, a processor communicates its nearest-neighbor one at a time. It is suitable for the parallel computer on a special homogeneous architecture such as the hyper-cube structure. With the DF method, a processor communicates all of the nearest-neighbors. It is superior for asynchronous 
processings in multi-port communications [10] associated with a heterogeneous distributed system. Therefore, we focus the DF method for load balancing in distributed systems on a general topology.

Recently, the optimal polynomial scheme (OPS) within a finite number of iterations has been proposed [3. The key-idea is to iteratively control polynomials for the calculation of diffusion flow by using the eigenvalues of a Laplacian. For parallel computers, it is an established method as a generalization of the conventional first order, second order, and Chebyshev schemes, and extended to the connection structure of a cartesian product of graphs 4]. However, there is a problem for the ordering of migration. It may cause a chain of load requirements for mutually related cycles, then a processor must be waited for a long time until the arrival of much load. Moreover, the calculations of all eigenvalues are necessary in advance for a topology. However, it is not negligible in distributed systems because the topology may be changed on the Internet, while to find the best topology is still open [2] (may be intractable).

In this paper, we propose an adaptive method according to initially assigned load. In this method induced from a quadratic programming (QP) problem [3 6] equivalent to solving the DF method, the optimal flow is obtained by a variation or perturbation on a cactus adaptively extended from a spanning tree (as a least connected component). The merits in computation and migration are that it can be directly calculated by the independency of each cycle in a cactus and the efficient message passings on a tree without cycles, and that the conditions of migration for the bottleneck edges are relaxed by bypasses on a cactus. Indeed, we show the proposed method is more efficient than the conventional tree walking algorithm (TWA) 7 through simulations for one-port and multi-port cases; The number of rounds is decreased under a certain ratio by the relaxation of the conditions for migration.

\section{Dynamic Load Balancing Problem}

In this section, we present a new problem setting for a dynamic load balancing in distributed computing on the Internet. Then, we point out that the DF method is equivalent to a $\mathrm{QP}$ problem, whose form is applied to the derivation of a variational or perturbative method on a cactus in the next section.

\subsection{Distributed System on the Internet}

Considering the differences of distributed computing to parallel computing: loose coupling, independence of processing elements, and heterogeneity [8], we define the load balancing problem in a network based on IP packet routings. To simplify the discussion, we assume that the performances of servers are the same.

- The amount of load is defined by the ratio of processes in ready state to the performance, which is measurable between the adjacent servers. We assume 
it is divisible as same in many literatures [2] [3] [4] 6] 10], because our application considered in this paper has very much data or many combination of parameters in independent processes.

- The connection between servers are logical on dynamic IP packet routings. We consider a computer-system on the Internet or in a WAN as Grid. However, the proposed method in this paper is applicable to a smaller system in a LAN.

- The connection structure is not restricted to regular ones, such as a ring, lattice, or homogeneous Cayley graph, but a heterogeneous general topology with both dense and sparse connections.

- Since long-distance connections may exists, we basically consider asynchronous local communications between servers.

Thus, processings are dominant than communication overheads in our application. Strictly speaking, the definition of load index is impossible without actually running, because it depends on many factors: not only on the response times for CPU, memory and I/O resources, but also on their communications or process granularity. It is known as "Key Issues in Dynamic Load Balancing" [10]. However, it is natural to consider the estimated values in a large problem with very much data or many combination of parameters.

\subsection{Equivalent QP Problem to the DF Method}

Although many schemes have been proposed for the DF method [2] 3] 6], it essentially result in solving a diffusion equation by a discrete Laplacian $L$ on a simple undirected graph $(V, E)$, whose vertices and edges are corresponding to the servers and connections, respectively.

The matrix-vector representation is

$$
L \mathbf{f}=\left[\begin{array}{ccccc}
\ldots & \ldots & -w_{e} & \ldots & \ldots \\
\vdots & \ddots & 0 & \vdots & \vdots \\
-w_{e} & 0 & \sum w_{e} & \vdots & \vdots \\
\vdots & \vdots & \vdots & \ddots & \vdots \\
\ldots & \ldots & \ldots & \ldots & \ldots
\end{array}\right]\left(\begin{array}{c}
f(1) \\
\vdots \\
f(u) \\
\vdots \\
f(|V|)
\end{array}\right)
$$

the $u$ th element is

$$
L f(u)=-\sum_{v \sim u} w_{e}(f(v)-f(u)),
$$

where $v \sim u$ denotes a set of the adjacent vertices to $u \in V, f(u)$ the amount of load at server $u, w_{e}=w_{\bar{e}}>0$ the weight of edge $(e ; u \rightarrow v$ or $\bar{e}: v \rightarrow u \in E$, which depends on only choosing the direction).

In this paper, $w_{e}$ is defined as "the stability of traffic for the migration between servers" (e.g. time-invariance measured by ping), not as an accelerative parameter in numerical schemes for the DF method. To discuss the characteristics of traffic, the conventional queuing theory based on independent Poisson 
arrival is insufficient, in even macroscopic phase transition, a statistical analysis such as Contact Process is needed for the self-similarity of packet density fluctuations 9. However, it is beyond our present scope. Thus, we don't consider the weights of communication efficiency for data transfer speed with delay or band-width, because it is microscopically unstable and indefinite in the dynamic routings.

Let us consider a diffusion equation:

$$
\frac{\partial \mathbf{f}}{\partial t}=-L \mathbf{f}
$$

It is converged to the solution

$$
\bar{f} \stackrel{\text { def }}{=} \frac{\sum_{u \in V} f(u)}{|V|},
$$

with monotone decreasing $\sum_{u \in V}(f(u)-\bar{f})^{2}$. It is also known that the total load $\sum_{u \in V} f(u)$ is conserved in (2) at any time.

Intuitively, the flow for migration is accumulated by the right side of (2) according to the difference of load between adjacent servers, and each flow on $e$ is enhanced to be much migration as larger $w_{e}$ (more stable) in the right side of (1).

On the other hand, as first order scheme, the difference equation for (2) is given by

$$
\mathbf{f}^{k}=(I-\Delta t L) \mathbf{f}^{k-1}=\underbrace{F \times \ldots \times F}_{k} \mathbf{f}^{0},
$$

where $F \stackrel{\text { def }}{=} I-\Delta t L, I$ denotes an unit matrix, $\mathbf{f}^{k}$ the load vector at $k$ th iteration, $\mathbf{f}^{0}$ initial load vector. We assume that the step-width $\Delta t$ satisfies $1 \leq \Delta t \times$ $\sum_{e \in E_{u}} w_{e}$, where $E_{u}$ denotes a set of edges connected to $u \in V$ [6]. Hereafter, we rewrite $w_{e}$ by $\Delta t \times w_{e}$.

Since the convergence of (4) is very slow, other schemes have been developed [3] [4] 6], in which we use that the equation (4) is equivalent to

$$
\begin{gathered}
y_{e}^{k-1}=-w_{e}\left(f^{k-1}(v)-f^{k-1}(u)\right), \\
z_{e}^{k}=z_{e}^{k-1}+y_{e}^{k-1}, \quad z_{e}^{0}=0, \\
f^{k}(u)=f^{k-1}(u)-\sum_{e \in E_{u}} y_{e}^{k-1},
\end{gathered}
$$

and also to the following QP problem [3] [6],

$$
\begin{aligned}
& \min \frac{1}{2} \mathbf{z}^{T} W^{-1} \mathbf{z}, \\
& \text { s.t. } B \mathbf{z}=\mathbf{f}^{0}-\overline{\mathbf{f}},
\end{aligned}
$$

where $W \stackrel{\text { def }}{=} \operatorname{diag}\left(w_{e}\right), B$ denotes the incidence matrix, $\mathbf{z}$ flow vector for the migration $\left(z_{e}\right.$ the flow on an edge $e \in E$, the positive value corresponds to the direction $), \overline{\mathbf{f}} \stackrel{\text { def }}{=}(\bar{f}, \ldots, \bar{f})$ the balancing solution vector of (31). 
In the QP problem, the balancing condition (6) includes a feasible solution with wastful flows on cycles, therefore the minimization (5) is needed. In other words, the equivalent DF method implicitly gives us the optimal solution as the global balancing. Note that (5) represents a cost with respect to the instability of migration. If we don't consider the weight (as all $w_{e}=1$ ), it becomes a simple cost for the no-wastful flow.

\subsection{Efficient Message Passing on a Tree}

When the connection structure is a spanning tree, the flow $z_{e}$ is directly obtained by efficient message passings, instead of the iterative calculations in such [2] 3] 44 6]. It is known as TWA [7, in which a feasible solution for (6) is the optimal, then $z_{e}$ is recursively calculated from leaves to the root as follows.

For a leaf $u: z_{e}=f(u)-\bar{f}$,

where $e \in E$ is an edge to the parent of $u \in V$.

For others: $z_{e^{\prime}}=f(v)-\bar{f}+\sum_{e} z_{e}$,

where $e^{\prime} \in E$ is an edge to the parent of $v \in V$, and $\{e\}$ in the summation is a set of edges from the children of $v$.

Although TWA is seemed to be a centralized control method, the communications are composed of the sending/receiving values of $z_{e}$ or the accumulated load (the individual values of load are not needed) to each parent and the broadcasting the value $\bar{f}$ from the root, its additional computation is only (3) at the root. Therefore, they can be performed by locally asynchronous communications and processings in a distributed manner.

\section{Adaptively Constructed Cactus}

In this section, for the load balancing, we propose an adaptive method on a cactus. A simple graph, whose each edge is contained in an exactly one cycle, is called "cactus." In the proposed method, we show that the optimal flow is directly obtained by a variation or perturbation for the QP problem, in which the flows of bottleneck edges are bypassed on a cactus. Consequently, the conditions of migration are relaxed, and the number of rounds is decreased as shown in the next section.

First, we consider a spanning tree as the least connection structure among servers. After applying TWA to it, at each vertex $u$, we find a bottleneck edge:

$$
e=\arg \max _{e \in E_{u}}\left\{\frac{\left|z_{e}\right|^{2}}{w_{e}}\right\},
$$

which depends on the initial load.

Next, in the connected edges to $u$, we find the pair $e^{\prime}$ which minimizes the cost for the extended QP problem by adding a bypass $e^{\prime \prime}$ as shown in Figure 1 The flow $z_{e^{\prime}}$ has the inverse direction of $z_{e}$ at $u$ (for input or output). 
We call the following approach "variation or perturbation," because the problem is extended to the minimization (5) on a cactus according to the initial load. Note that the balancing condition (6) still holds due to the bypass modifications.

For the bypass flow $\Delta z$ on $e^{\prime \prime}$, the variation of cost in (5) is given by

$$
\delta C(\Delta z) \stackrel{\text { def }}{=} \frac{\left(z_{e}-\Delta z\right)^{2}}{w_{e}}+\frac{\left(z_{e^{\prime}}-\Delta z\right)^{2}}{w_{e^{\prime}}}+\frac{\Delta z^{2}}{w_{e^{\prime \prime}}}-\left(\frac{z_{e}^{2}}{w_{e}}+\frac{z_{e^{\prime}}^{2}}{w_{e^{\prime}}}\right) .
$$

The most decreasing is obtained at the extreme point $\frac{\partial(\delta C)}{\partial(\Delta z)}=0$. Since each cycle on a cactus is independent, we can easily derive the solution

$$
\Delta z_{o p t}=\frac{w_{e^{\prime}} w_{e^{\prime \prime}} z_{e}+w_{e} w_{e^{\prime \prime}} z_{e^{\prime}}}{w_{e^{\prime}} w_{e^{\prime \prime}}+w_{e} w_{e^{\prime \prime}}+w_{e} w_{e^{\prime}}}>0 .
$$

From this, we also derive $\delta C\left(\Delta z_{\text {opt }}\right)<0$; the cost is always decreased by adding bypasses on a cactus.

Thus, we can obtain the optimal solution for the larger QP problem (equivalent to solving the DF method) on an adaptively extended cactus by applying only local modifications, because each cycle is independent; If there are common edges on cycles, iterative calculations are necessary. We summarize the adaptive method on a cactus as follows. The communication complexity is $O(|V|)$.

$$
<\text { Distributed Algorithm on a Cactus > }
$$

Step 0. At the trriger from a server with heavy load, the following processes for the global balancing are initiated in run-time.

Step 1. Construct a minimum spanning tree (MST) by applying a distributed algorithm e.g. 5]. Here, the distance may be based on geographical locations of servers, or on the averaged communication delay.

Step 2. Calculate the flow by applying TWA to it.

Step 3. Find a bottleneck edge and the candidate of bypass at each vertex, independently. To construct a cactus (Figure 2), the candidates of bypass are mutually excluded by time-stamps or other appropriate criterions.

For the fixed bypass, calculate the modified flows $z_{e}-\Delta z_{o p t}, z_{e^{\prime}}-\Delta z_{o p t}$, and $\Delta z_{\text {opt }}$ by (7).

Step 4. Asynchronously migrate it between servers, as soon as possible (first come, first served) in a locally distributed manner.

Altough the above discussion is devoted to a ternary cycle, it is quite same for other cycles: quaternary or longer cycles. However, the ternary is practically better in the following reasons.

- The mutual exclusion is restricted in the alternative combination of triangles. If we consider longer cycles, it may be intractable that many edges are complicatedly related.

- Each server can directly communicate to the nearest-neighbors. While, for longer cycles, it must pass the information $z_{e}$ or $w_{e}$ through intermediators.

- Both ends of a bypass edge are probably close in the geographical locations. 


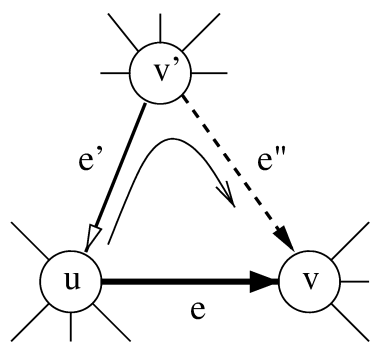

Fig. 1. Bypass for a bottleneck edge $e$.

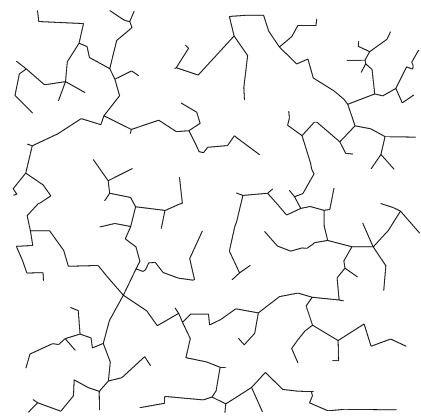

Fig. 3. $\operatorname{MST}(|V|=300)$.

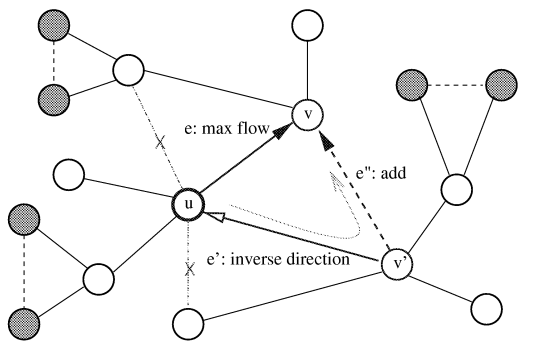

Fig. 2. Mutual exclusion among three triangles for constructing a cactus. The other triangles far from them have norelation.

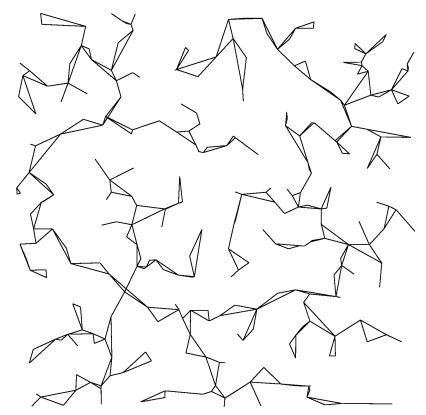

Fig. 4. Ternary cactus $(|V|=300)$.

\section{Simulation Results}

We investigate the effect of the relaxed conditions for migration by bypasses on an adaptively constructed cactus according to the initial load. Since the migration is a process in Step 4 after the calculation of flow, the effect is common and independent of any schemes (even if we apply other schemes such as OPS [3]), but depends on the initial load and the topology.

To set a topology among servers and investigate the general properties in our method, we first construct graphs with sizes $|V|=100,200,300,400,500$, whose vertices are randomly located. In addition, we set the weight $w_{e}$ on the complete graph and the initial load $f(u)$ at each vetrex by $(0.5,1)$ and $(0,200)$ uniformly random numbers, respectively. In this first setting, we consider the complete graph to only prepare for the extension to a cactus from the MST. After fixing a topology, the vertex and edge are corresponding to a server and connection for the communication. Remenber that $w_{e}$ represents the stability of migration between servers. We also set the time-stamps by $(0,1)$ uniformly 
random numbers. An example of the MST is shown in Figure 3, and a cactus constructed by the previous algorithm is shown in Figure 4

We verify the effect of bypasses on a cactus. In our simulations, to simply the discussion, we assume that there exist a constant interval between the activations for the migration. Because a server with less load than $z_{e}$ must be waited until the required load is arrived, even if each server can asynchronously activate it. We call the timing for migration "round," which is not depended on the amount of flow and the routing. At each round, the edges which satisfy the condition of migration become active. Note that the asynchronous property is included in the random time-stamps. Strictly speaking, since the activation depends on the data transfer speed, the delay by traffic congestion, and the application (the amount of data for migration), it is an underestimation. In other words, a real system takes much more times than the number of rounds $\times$ a constant interval.

We investigate the minimum, maximum, and average values of the rounds in ten trials for each size of graphs. In the multi-ports, the migrations can be simultaneously activated at a vertex. The difference between the minimum and maximum is due to whether fitting or unfitting of the initial load for the topology; in a case, the load may be migrated through many and long paths on the MST or cactus. The average rounds are shown in Figure 5. By the bypasses on a cactus, the conditions of migration are relaxed, then the rounds are decreased; the ratio (of the rounds on a cactus to that on the MST) is about 2/3 as shown in Figures 6] and 7 The result for the multi-ports is about $10 \%$ improved than that for the one-port.

Figures 8 and 9 show the ratio of the costs with respect to the instability of migration. By the bypasses on a cactus, it is also decreased to about the half $(0.4 \sim 0.5)$. Moreover, the average efficiency: active edges for the migration per rounds is about twice of that in the MST. Through the simulations, we have found that the bypasses on a cactus are constantly $36 \%$ added for all of sizes $|V|$ and initial load in ten trials. It suggests that there exist a something of general law in constructing a ternary cactus from any spanning tree.

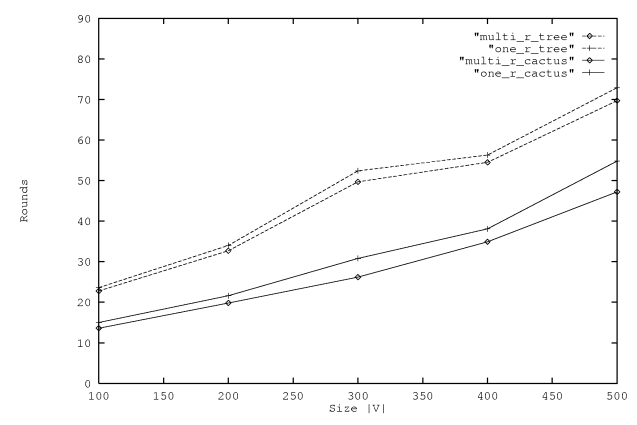

Fig. 5. Average rounds for the multi-ports $\diamond$ and one-port + cases. The solid and dashed lines are corresponding to the results for a cactus and the MST, respectively. 


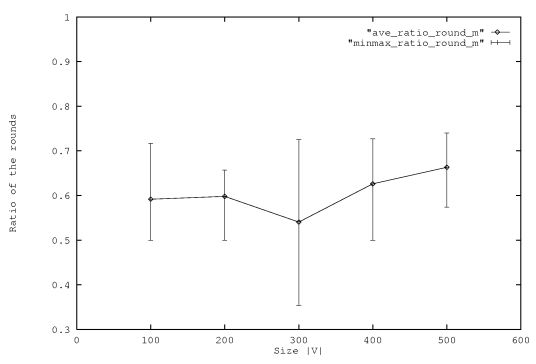

Fig. 6. Ratio of the rounds (multiports).

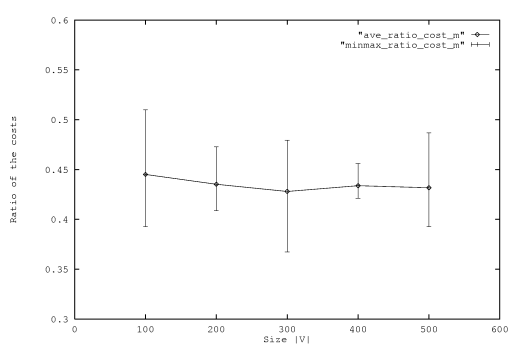

Fig. 8. Ratio of the costs (multi-ports).

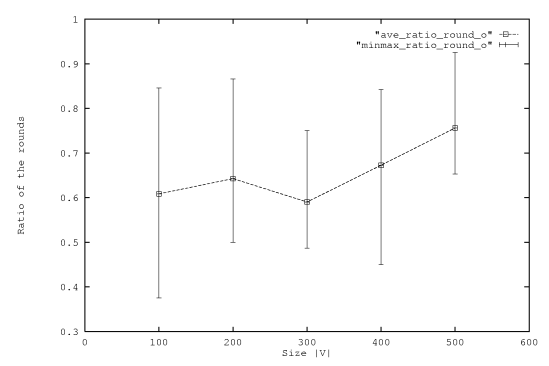

Fig. 7. Ratio of the rounds (one-port).

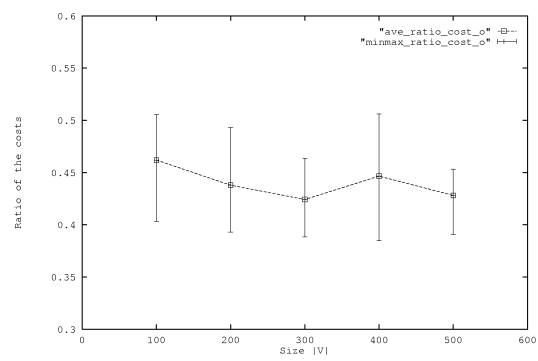

Fig. 9. Ratio of the costs (one-port).

\section{Conclusion}

We have considered the dynamic load balancing problem for servers on the Internet as Grid, based on the differences of distributed computing to parallel computing: loose coupling, independence of processings, and heterogeneity. In general, the efficiency for load balancing depends not only on calculation schemes for the migration flow but also on heterogeneous topologies of the connection between servers. However, to find the optimal topology is intractable in the state-of-the-art.

In this paper, we have proposed an adaptive method according to initially assigned load, and presented it as a distributed algorithm based on only local communications and asynchronous processings. The method has been induced from a QP problem equivalent to solving the DF method, in which the optimal flow is obtained by using local variational or perturbative computations, and the conditions of migration for the bottleneck edges are relaxed by the bypasses on a cactus adaptively extended from the MST. 
Simulation results have shown that the number of rounds for the migration is decreased under $2 / 3$ for the conventional TWA, and that the cost with respect to the instability of migration is also decreased in about the half. These results are underestimations, however, not depended on any schemes and implementation technologies. Thus, the adaptively constructed cactus is considered as a practically efficient topology, in addition, suitable for dynamically reconstructive distributed systems (with flexible connections and changes of servers). The implementation and evaluation on real systems are further studies, e.g. for parameter search in scientific computations or cooperative Web crawlers.

\section{References}

1. T. Bourke. Server Load Balancing, O'Reilly, 2001.

2. T. Decker, B. Monien, and R. Preis. "Towards Optimal Load Balancing Topologies," A. Bode et al. (Eds): Euro-Par2000, LNCS 1900, pp. 277-287, 2000.

3. R. Diekmann, A. Frommer, and B. Monien. "Efficient Schemes for Nearest Neighbor Load Balancing," Parallel Computing, Vol. 25, pp. 789-812, 1999.

4. R. Elsässer, A. Frommer, B. Monien, and R. Preis. "Optimal and AlternatingDirection Load Balancing Schemes," P. Amestoy et al. (Eds.): Euro-Par'99, LNCS, 1685, pp. 280-290, 1999.

5. R. Gallager, P. Humblet, and P. Spira. "A Distributed Algorithm for Minimun Weight Spanning Trees," ACM Trans. on Prog. Lang. and Systems, Vol. 5, No. 1, pp. 66-77, 1983.

6. Y.F. Hu, R.J. Blake. "An Improved Diffusion Algorithm for Dynamic Load Balancing," Parallel Computing, Vol. 25, pp. 417-444, 1999.

7. W. Shu, and M.Y. Wu. "Runtime Incremental Parallel Scheduling on Distributed Memory Computers," IEEE Trans. on Parallel and Distributed Sysytems, vol. 7, no. 6, pp. 637-649, 1996.

8. V.S. Sunderam, and G.A. Geist. "Heterogeneous Parallel and Distributed Computing," Parallel Computing, Vol. 25, pp. 1699-1721, 1999.

9. M. Takayasu, A.Yu. Tretyakov, K. Fukuda, and H. Takayasu. "Phase Transition and $1 / f$ noise in the Internet Packet Transport," D.E. Wolf et al. (Eds): Traffic and Granular Flow '97, Springer Singapore, pp. 57-74, 1998.

10. C. Xu, and F.C.M. Lau. Load Balancing in Parallel Computers -Theory and Practice-, Kluwer Academic Publishers, 1997. 